\title{
Successful Non-Operative Management of Multi-Trauma Patient Suffering from Multiple Intra-Abdominal Injuries - A Case Report
}

\author{
Georgios Theodoros Liagkos, $M D^{1}$, Christos Chouliaras, $M D^{1 *}$, Aris Papadopoulos, $M D^{1}$ \\ and Constantine Vagianos, $M^{2}$
}

${ }^{1}$ Department of Surgery, Nikaia General Hospital, Nikaia, Piraeus, Greece

${ }^{2}$ Propedeutic Department of Surgery, Laikon Hospital, National \& Kapodestrian University of Athens, Athens, Greece

*Corresponding author: Christos Chouliaras, M.D, Department of Surgery, Nikaia General Hospital, Nikaia, Piraeus, Greece

\begin{abstract}
Trauma represents the main cause of death among people under the age of 35 worldwide. Until the 90 s, the operative management $(\mathrm{OM})$ was the golden standard of treatment for trauma patients with abdominal bleeding. Over the last few decades, though, a shift has been made from operative to non-operative management (NOM) in haemodynamically stable trauma patients with findings of recent intra-abdominal bleeding and no signs of peritonitis. However, NOM may be particularly challenging in patients with multi-abdominal organ injuries since evaluation and total treatment may be exceptionally difficult. We report the case of a 25-year-old patient with blunt abdominal trauma (BAT) suffering multiple injuries of the spleen and liver, pelvic fracture and right femur fracture who has been successfully treated through NOM.
\end{abstract}

\section{Keywords}

Non-operative management, Multi-organ injuries, Hemodynamic stability

\section{Introduction}

Trauma is the leading cause of death in people under the age of 35 worldwide [1]. Abdominal injuries occur in $31 \%$ of polytrauma patients, among whom $13 \%$ suffer liver, $16 \%$ spleen and $28 \%$ pelvic injuries [2]. Selective non-operative management of significant abdominal injuries represents one of the most important changes in the trauma patient care over the last decades [3]. Non-operative management of blunt traumatic injuries is well-established, and strategies based on focus assessment with sonography for trauma (FAST) scan, CT scan diagnosis and the hemodynamic stability of the patient are now being widely used in the treatment of solid organ injury, including liver, spleen, kidneys as well as pelvic injuries. Selective non-operative management has become the standard of care in BAT including severe solid organ injuries [4].

Now a days, trauma patients with signs of peritonitis or hemodynamic instability should be urgently taken for laparotomy provided that Emergent Department ultrasound investigation has revealed intra-abdominal blood [5]. Whole-body contrast enhanced Computed Tomography (WBCT) is an important adjunct in trauma care that is part of the standard protocol in initial assessment of stable or post-resuscitation stabilized trauma patients scheduled for NOM [6]. CT is the default imaging of choice in severely injured patients as its high diagnostic accuracy leads to the detection of injuries and can accurately identify the grade of injury [7]. Blunt abdominal trauma may not occur in isolation and may be associated with significant extra-abdominal injuries, many of which will determine the overall outcome. When following the NOM protocols, laparotomy is required in less than $10 \%$ of trauma patients with recent abdominal bleeding [8]. The presence of multiple solid organ injuries should not preclude the use of NOM. Despite higher failure rate of NOM in multiple solid organ injuries, it can still be chosen as the best treatment in most haemodynamically stable patients without peritoneal signs. Thus, extra caution should be shown so as not to significantly increase morbididy $[9,10]$. Poly-

\footnotetext{
Citation: Liagkos GT, Chouliaras C, Papadopoulos A, Vagianos C (2019) Successful Non-Operative Management of Multi-Trauma Patient Suffering from Multiple Intra-Abdominal Injuries - A Case Report. Trauma Cases Rev 5:077. doi.org/10.23937/2469-5777/1510077 Accepted: September 12, 2019: Published: September 14, 2019

Copyright: (c) 2019 Liagkos GT et al. This is an open-access article distributed under the terms of the Creative Commons Attribution License, which permits unrestricted use, distribution, and reproduction in any medium, provided the original author and source are credited.
} 
trauma patients represent the ultimate challenge for a trauma surgeon due to their broad spectrum of injured organs and clinical scenarios [11].

\section{Case Report}

A 25-year-old male was transferred to the Emergency Department of the tertiary Regional Hospital of Nikea after a high-energy motor-vehicle collision. Initial management was based on the protocols of Advanced Trauma Life Support (ATLS). At first evaluation, he was haemodynamically unstable with sinus tachycardia and $80 \mathrm{mmHg}$ systolic blood pressure requiring bolus infusion. The patient responded rapidly to the infusion of 1 It of Ringer's solution and his hemodynamic condition stabilized having a heart rate of 90 and 80 $\mathrm{mmHg}$ mean arterial pressure. At physical exam, he had a mild left upper quadrant abdominal pain without signs of peritonitis and mild pelvic tenderness without instability. His airway, lungs and brain presented no clinical radiological damage, GCS 14/15. Further evaluation for injuries showed a complete displaced

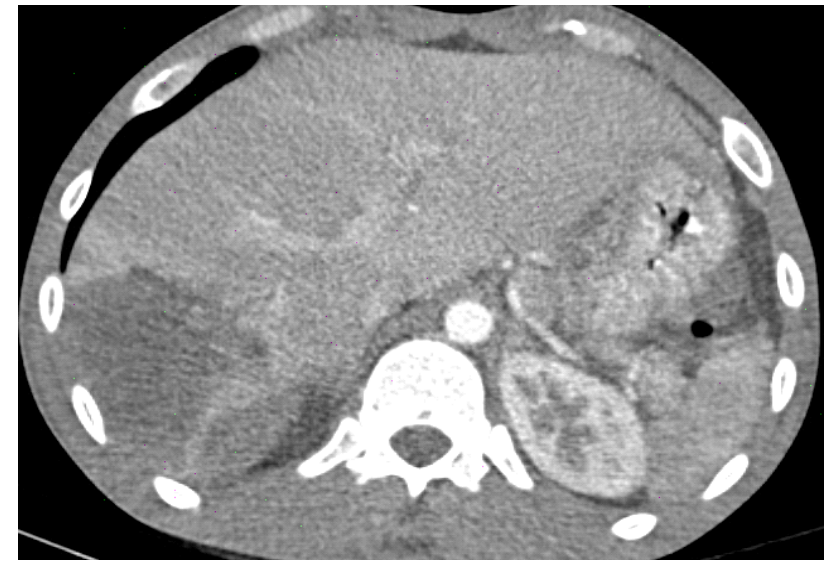

Figure 1: The contrast computed tomography (CT) scan demonstrates a large parenchymal hematoma in segment 6 and 7 of the liver with no active extravasation and an intraparenchymal hematoma of the spleen. right femoral shaft fracture causing deformity. Inline longitudinal traction was applied, realigning the extremity and maintaining limb perfusion. He reported no significant past medical history and denied any medication. A FAST scan was carried out that showed perisplenic and perihepatic fluid. Since he remained stable, a multi-detector whole-body contrast-enhanced computed tomography was performed revealing an American Association for the Surgery of Trauma (AAST) grade II splenic injury (Figure 1), a AAST grade II liver injury (Figure 1) and a non-displayed fracture of right ischiopubic ramus with the active contrast extravasation indicating arterial bleeding (Figure 2).

The patient was re-evaluated and since he remained stable, NOM was decided. In order to control active arterial bleeding in the pelvis, interventional radiology treatment was considered. Regarding the associated concomitant spleen, liver and femur injuries in addition to ongoing pelvic bleeding that could lead to a subsequent hemodynamic instability, we proceeded to angiography aiming to trans-arterial embolization. Other determinants to lead the patient to angiography were the continuous need for a resuscitation after a "fast responder" status and the non 24-hour accessibility of our interventional radiology department. A 5F Cobra-type (Cook, Bloomington, Indiana) angiography catheter was introduced and a pelvic aortography was performed, followed by a right internal iliac angiogram. Selective angiography showed an active bleeding from a branch of the internal pudendal artery. A microcatheter (Asahi Intecc, Seto, Japan) was needed for further advancement into the artery. The vessel was successfully embolized supra-selectively by infusing Micro-coils. The choice of materials were influenced by current availability. On check angiography, the bleeding was stopped and the patient remained haemodynamically stable (Figure 3 ). The duration of the procedure was 80 minutes. The procedure was well-tolerated, successful and without any

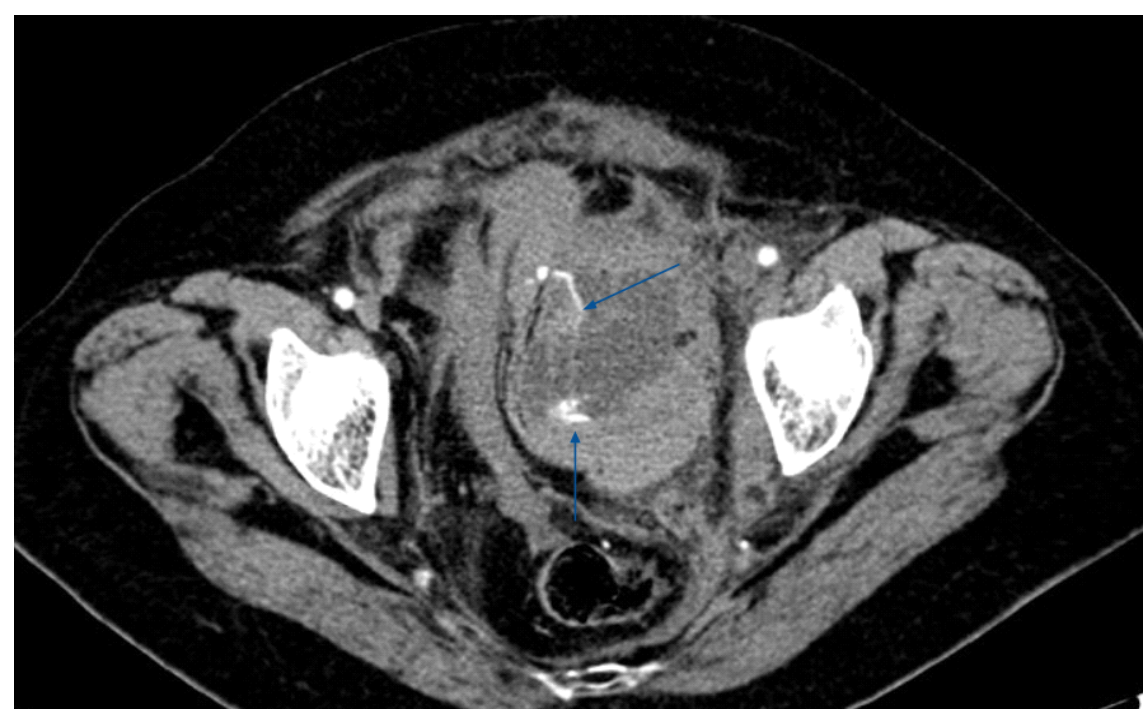

Figure 2: CT reveals extravasation of contrast material within a pelvic hematoma. 


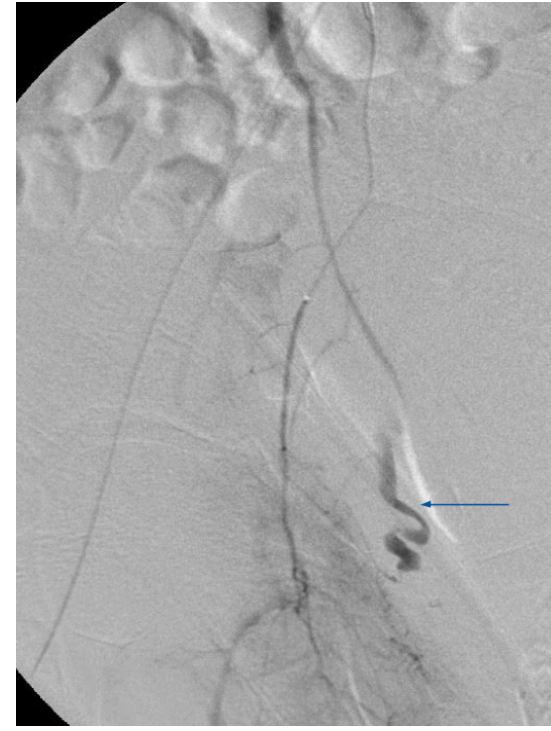

a

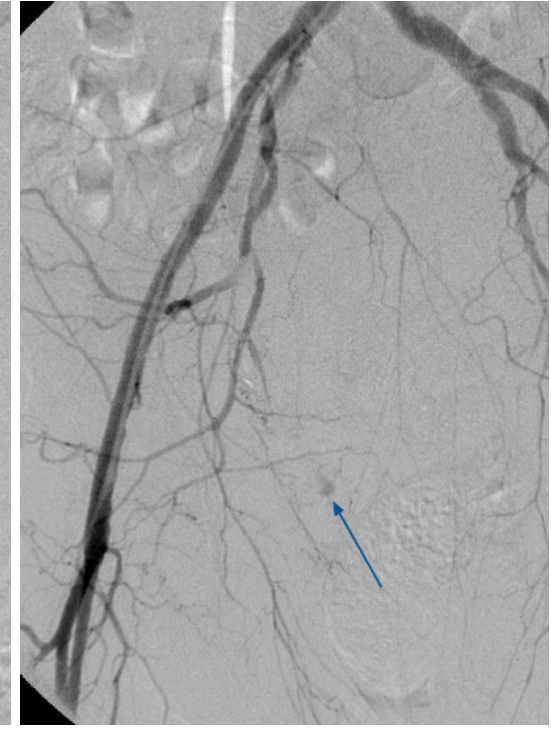

b

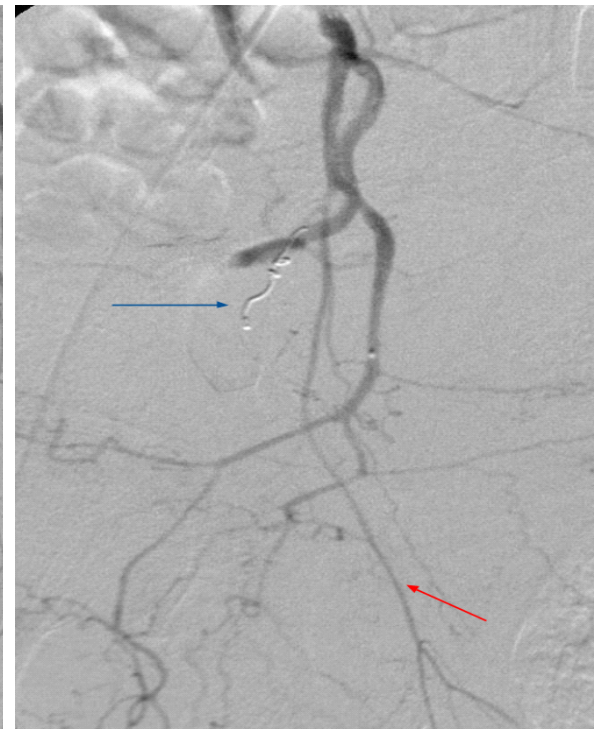

$\mathrm{C}$

Figure 3: $a, b)$ Transfemoral digital artery angiogram of right pelvic side shows active bleeding from a branch of interior pudendal artery; c) Proximal transcatheter selective embolization of the vessel with spiral coils was achieved and the bleeding was stopped.

\section{complications.}

The patient, remaining in acceptable hemodynamic condition, was admitted to the intensive care unit immediately after the procedure for close observation, appropriate resuscitation, serial clinical examinations and blood tests. The right extremity was set in skin traction. He received low-weight molecular heparin for deepvein thrombosis prophylaxis on post-trauma day (PTD) 2. Definitive treatment for right complete femoral shaft fracture involving surgical stabilization on PTD 4 after a repeated computed tomography, which demonstrated stable abdominal injuries, and after a triplex ultrasound examination, which ruled out any vascular injury was performed by an orthopedic surgeon. The fractured was treated with intramedullary nailing.

During hospitalization, the patient remained asymptomatic, haemodynamically stable and normal, receiving a total of 9 blood products ( 3 packed red-blood cell units, 3 fresh frozen plasma units, and 3 platelets units) according to his demands. He was transferred to the Surgical Department on PTD 7 and was discharged on PTD 11 having 25.8 haematocrit and hemoglobin of 9.1 and prescribed appropriate low weight molecular heparine as thromboembolism prophylaxis. The patient tolerated well the recovery period with extensive physical therapy. After 10 weeks, the patient started bearing weight and mobilizing and gradually regained fully walking capacity. The patient showed no associated complication in the follow-up one year post-injury, is still doing well and has returned to sports and physical activity.

\section{Discussion}

The management of trauma patients with multiple organ injuries combined with pelvic injuries poses significant diagnostic and treatment challenges. It demands careful evaluation and prompt recognition of the hemodynamic instability and the response to resuscitation. Misjudgment of these parameters may be catastrophic while the right evaluation is of great importance and dictates the appropriate management. Multiple injuries are not contraindication to NOM and these patients should also be considered to such an approach. Further management depends on the haemodynamic stability of the patient. In our case, the patient responded to resuscitation rapidly and remained stable after his injuries had been identified and staged by CT scanning.

Non-operative treatment may even be considered in multiple severe injuries as long as the patient is haemodynamically stable and facilities for hemodynamic monitoring and emergency exploration are available if required. Non-operative treatment should be attempted in high dependency/intensive care environment, including close clinical observation and possibility for regular laboratory and imaging assays. Therefore, management may be modified according to the needs of the patient necessitating access to blood and blood products, diagnostics and rapidly available invasive procedures including angiography and angioembolization [12]. Only under these conditions, should NOM be reliably applied. However, NOM in haemodynamically normal patients with several intra-abdominal organ injuries is related to prolonged intensive care unit and hospital stay, complications and more often failure of NOM when compared to injury of an isolated organ [13]. It should be overemphasized that this type of management should be applied only by experienced surgeons in trauma centers bearing facilities for early detection of 


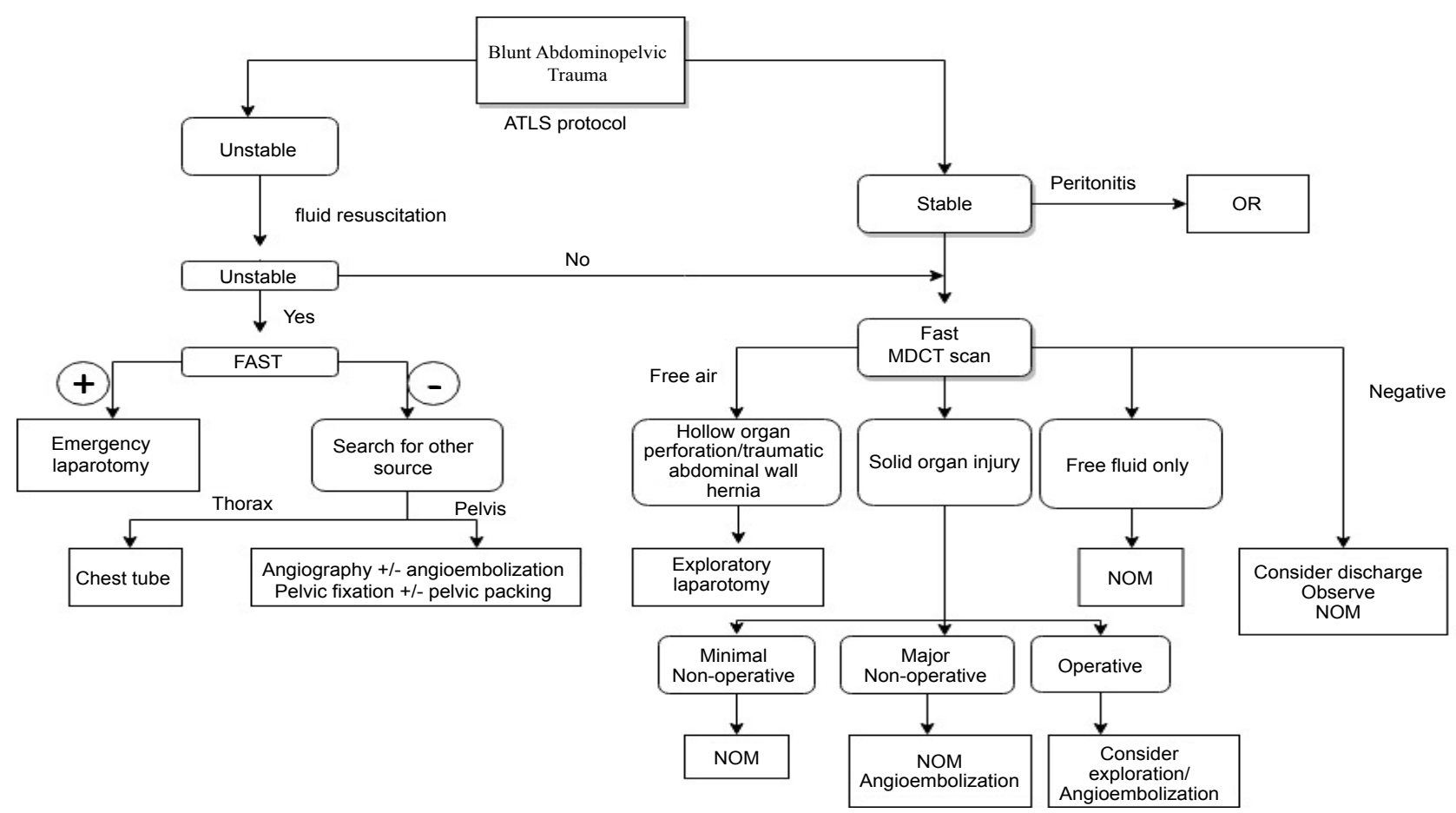

Figure 4: Diagnostic and therapeutic algorithm for patients with blunt abdominopelvic trauma.

complications and prompt conversion to often demanding surgical strategies [8]. Notably, predictive factors of successful non-operative management of patients with intra-peritoneal bleeding following blunt abdominal trauma are the shock index, the systolic blood pressure on arrival, the base deficit and the hemoglobin drop within the first 12 hours of admission [14]. Advances in invasive radiology allow higher rates of success, achieving bleeding control by embolization of abdominal and pelvic vessels [15]. As mentioned above, the liver and spleen, representing the most commonly injured organs, as well as pelvic injuries could benefit from NOM. NOM and angioembolization is applied in liver trauma patients presented with multiple injuries and also with high grade liver injuries with a high overall NOM and angioembolization success rate $[16,17]$. Therapeutic endovascular embolization is an extension of NOM which not only achieves hemostasis at the site of vascular injury or difficult to access surgical sites, but also controls rebleeding after surgery [18]. Pelvic angioembolization is the most effective intervention for the management of hemorrhage associated with pelvic fracture in both hemodynamic stable and unstable patients and can be used in the setting of concomitant intra-abdominal injury [19]. The indications for angiography embolization after pelvic fracture are controversial. It has been suggested that the presence of extravasation blush or pelvic hematoma on computed tomography are predictors of active and significant bleeding respectively [20]. Accordingly, selective embolization after localization of the bleeding vessel by arteriography was successfully performed to our patient. Angiography/embolization is a minimally invasive procedure with excellent outcomes in hemorrhagic complications of pelvic fractures.
Furthermore, NOM reduces blood transfusion requirement, morbidity, mortality, and the incidence of unnecessary laparotomies. Even cases with multiple abdominal injuries can be successfully managed by applying NOM regardless of the grade of injury, as long as they follow the principles of treatment described earlier [21].

In conclusion, the new understanding of trauma management, resuscitation, coagulation and in correlation with advanced radiologic and embolization techniques has allowed a shift towards non-operative management of trauma patients $[22,23]$. We propose a diagnostic and therapeutic algorithm for patients with blunt abdominopelvic trauma, that represents a safe and sensible approach to the evaluation of these patients and a pathway to NOM treatment (Figure 4).

This case supports the safety of NOM in a polytrauma patient. We feel that this type of management is only appropriate for highly selected patients in combination with good clinical assessment by an experienced surgical team in the environment of a highly-specialized trauma center.

\section{Acknowledgment}

\section{Conflict of interest}

There are no conflicts of interest.

\section{References}

1. Søreide K (2009) Epidemiology of major trauma. Br J Surg 96: 697-698.

2. Beuran M, Negoi I, Paun S, Runcanu A, Venter D, et al. (2010) Selective nonoperative management of solid abdominal visceral lesions. Chirurgia (Bucur) 105: 317-326. 
3. Luke PH, Leene K (2009) Abdominal trauma: From operative to no-operative management. Int J care Inj 40: S62-S68.

4. Stawicki SP (2017) Trends in nonoperative management of traumatic injuries - A synopsis. Int J Crit IIIn Inj Sci 7: 38-57.

5. Malhotra AK, Ivatury RR, Latifi R (2002) Blunt abdominal trauma: Evaluation and indications for laparotomy. Scand J Surg 91: 52-57.

6. Huber-Wagner S, Kanz KG, Hanschen M, van Griensven M, Biberthaler P, et al. (2018) Whole-body computed tomography in severely injured patients. Curr Opin Crit Care 24: 55-61.

7. Çorbacıoğlu ŞK, Aksel G (2018) Whole body computed tomography in multi trauma patients: Review of the current literature. Turk J Emerg Med 18: 142-147.

8. Howes N, Walker T, Allorto NL, Oosthuizen GV, Clarke DL (2012) Laparotomy for blunt abdominal trauma in a civilian trauma service. S Afr J Surg 50: 30-32.

9. Yanar H, Ertekin C, Taviloglu K, Kabay B, Bakkaloglu $\mathrm{H}$, et al. (2008) Nonoperative treatment of multiple intraabdominal solid organ injury after blunt abdominal trauma. J Trauma 64: 943-948.

10. Sartorelli KH, Frumiento C, Rogers FB, Osler TM (2000) Nonoperative management of hepatic, splenic, and renal injuries in adults with multiple injuries. J Trauma 49: 56-61.

11. Gaspar B, Negoi I, Paun S, Hostiuc S, Ganescu R, et al. (2014) Selective nonoperative management of abdominal injuries in polytrauma patients: A protocol only for experienced trauma centers. Maedica (Buchar) 9: 168-172.

12. Coccolini F, Montori G, Catena F, Kluger Y, Biffl W, et al (2017) Splenic trauma: WSES classification and guidelines for adult and pediatric patients. World J Emerg Surg 12: 40.

13. Teuben MPJ, Spijkerman R, Blokhuis TJ, Pfeifer R, Teuber $H$, et al. (2018) Safety of selective nonoperative management for blunt splenic trauma: The impact of concomitant injuries. Patient Saf Surg 12: 32.

14. Heidar A, Ravanfar P, Namazi G, Nikseresht T, Niakan H
(2014) Determinants of successful non-operative management of intraperitoneal bleeding following blunt abdominal trauma. Bull Emerg Trauma 2: 125-129.

15. Petrone $P$, Anduaga Pena MF, Servide Staffolani MJ, Brathwaite C, Axelrad, et al. (2017) Evolution of the treatment of splenic injuries: From surgery to non-operative management. Cir Esp 95: 420-427.

16. Brillantino A, lacobellis F, Festa $P$, Mottola A, Acampora C, et al. (2019) Non-operative management of blunt liver trauma: Safety, efficacy and complications of a standardized treatment protocol. Bull Emerg Trauma 7: 49-54.

17. Van der Wilden GM, Velmahos GC, Emhoff T (2012) Successful nonoperative management of the most severe blunt liver injuries: A multicenter study of the research consortium of new England centers for trauma. Arch Surg 147: 423-428.

18. Singh A, Kumar A, Kumar P, Kumar S, Gamanagatti $S$ (2017) "Beyond saving lives": Current perspectives of interventional radiology in trauma. World J Radiol 9: 155-177.

19. Bramos A, Velmahos GC, Butt UM, Fikry K, Smith RM, et al. (2011) Predictors of bleeding from stable pelvic fractures. Arch Surg 146: 407-411.

20. Salcedo ES, Brown IE, Corwin MT, Galante JM (2016) Pelvic angioembolization in trauma - Indications and outcomes. Int J Surg 33: 231-236.

21. Liagkos GT, Spyropoulos C, Tsourouflis G, Papadopoulos A, loannides $P$, et al. (2018) Successful non-operative management of blunt abdominal trauma in highly selective cases: A safe and effective choice. Ulus Travma Acil Cerrahi Derg 24: 104-109.

22. Ptohis ND, Charalampopoulos G, Abou Ali AN, Avgerinos ED, Mousogianni I, et al. (2017) Contemporary role of embolization of solid organ and pelvic injuries in polytrauma patients. Front Surg 4: 43.

23. van der Vlies $\mathrm{CH}$, Olthof DC, Gaakeer M, Ponsen $\mathrm{KJ}$, van Delden OM, et al. (2011) Changing patterns in diagnostic strategies and the treatment of blunt injury to solid abdominal organs. Int J Emerg Med 4: 47. 